\title{
miRNA Involvement in Angiogenesis in Age-related Macular
}

\section{Degeneration}

Lei Wang ${ }^{1}$, Amy Yi Wei Lee ${ }^{2}$, Jonathan P Wigg ${ }^{3}$, Hitesh Peshavariya ${ }^{3}$, Ping Liu ${ }^{1 *}$, Hong Zhang $^{1,3^{*}}$

1 Eye Hospital, Harbin Medical University, 23 Youzheng Street, Nangang District, Harbin, Heilong-jiang Province, China

2 Department of Pharmacology and Therapeutics, University of Melbourne, Australia; Drug Delivery Unit, Centre for Eye Research Australia

3 Centre for Eye Research Australia, University of Melbourne, Royal Victorian Eye and Ear Hospital, 32 Gisborne Street, East Melbourne VIC 3002, Australia

*Correspondence author:

Hong Zhang, T: +61 39288 3721; F: +61 9662 3859; email: hong.zhang@ unimelb.edu.au

Ping Liu, T: +86 451 85553927; F: +86 45185553921; email: pingliu53@126.com 


\begin{abstract}
Age-related macular degeneration (AMD) is the leading cause of blindness in the elderly. Late stage AMD is characterised by choroidal neovascularization (CNV). miR-93 appears to play a role in regulating vascular endothelial growth factor-A (VEGF-A) a known factor involved in neovascularisation. Understandings its biological significance might enable development of therapeutic interventions for diseases like AMD. We aimed to determine the role of miR-93 in AMD using a laser-induced CNV mouse model. CNV was induced by laser photocoagulation in C57BL/6 mice. The CNV mice were transfected with scrambled miR or miR-93 mimic. The treatment effect was assessed by fundus photography and fluorescein angiography and confirmed by choroidal flatmount. The expression of miR-93 and VEGF-A in ocular tissues were analysed by qPCR and western blot. The overexpression effects of miR-93 were also proved on Human microvascular endothelial cells (HMECs). Significantly decreased expression of miR-93 was observed by qPCR analysis in CNV mice compared to untreated mice $(\mathrm{p}<0.05)$. VEGF-A mRNA and protein expression were upregulated with $\mathrm{CNV}$; these changes were ameliorated by restoration of miR-93 $(\mathrm{p}<0.05)$. CNV was reduced after miR-93 transfection. Transfection of miR-93 reduced the proliferation of HMECs
\end{abstract}


$(\mathrm{p}<0.01)$, but no significant changes were observed in 2D-capillary-like tube formation $(p>0.05)$ and migration $(p>0.05)$ compared with that in the untreated cells. miR-93 has been shown to be a negative modulator of angiogenesis in the eye. All together these results high lights the therapeutic potential of miR-93 suggests that it may contribute as a putative therapeutic target for AMD in humans.

Key words: miR-93, Age-related-macular-degeneration, choroidal neovascularization, VEGF-A, HMEC

\section{Introduction}

Age-related macular degeneration (AMD) is a common ocular condition and the leading cause of blindness in the elderly and profoundly disrupts the individuals quality of life. AMD has a worldwide prevalence of about $8.7 \%$, which poses a significant burden on the global healthcare system ${ }^{[35]}$. The progression of this disease varies from one person to another as they age. Exudative or 'wet type' AMD accounts for $10 \%$ of all AMD cases ${ }^{[14]}$ and is choroidal neovascularization $(\mathrm{CNV})$ - an aberrant growth of blood vessels in the macula from the choroid into the subretinal space. These vessels are prone to leakage of fluid and blood below the macula, leading to the activation of inflammatory cascades, subsequent irreversible fibrotic scarring and retinal detachment, and causes permanent central visual

impairment ${ }^{[2]}$. Unfortunately, currently there is no 'early detection' biomarker for AMD is available, and the complex multifactorial pathogenesis of AMD is not yet fully understood. 
Vascular endothelial growth factor (VEGF), one of the most potent stimulators of angiogenesis, has been implicated in CNV formation and is the target of the majority of AMD therapeutics. Extensive studies have demonstrated that blocking the production of VEGF or its signaling pathways can suppress CNV in the eye ${ }^{[17]}$. Clinically, VEGF antagonists have been used widely for preventing further degeneration of the retina and stabilizing visual acuity in AMD patients ${ }^{[32]}$. However, the administration of anti-VEGF therapy has led to some severe side effects such as stroke ${ }^{[30]}$. Moreover, VEGF inhibitors have shown to be ineffective in a subset of AMD patients ${ }^{[33]}$. Hence, other options for treating this disease need to be explored.

miRNAs are short RNA molecules, approximately 20-22 nucleotides long, which regulate gene expression posttranscriptionally, mainly through translational repression or mRNA degradation $^{[7]}$. Specific miRNAs have shown to be abundant in the vascular system and play an extensive role in the regulation of angiogenesis ${ }^{[3]}$. As pathogenic neovascularisation plays a key role in the late stage of AMD, further exploration into the function of miRNAs may contribute to prospective treatments for this condition. At present, both in vitro and in vivo studies have revealed that a number of miRNAs are associated with similar pathogenic mechanisms of $\mathrm{AMD}^{[31]}$. It was found that numerous miRNAs such as miR-21, miR-126, miR130a and miR-221 are involved in ocular angiogenic processes ${ }^{[5]}$.

miR-93, a paralog of the miR-17-92 cluster, is predicted to target the $3^{\prime}$-untranslated region (UTR) of VEGF-A and is implicated in angiogenesis with controversial roles. In one study by Fang et al., miR-93 was found to promote angiogenesis and enhance tumor growth ${ }^{[11]}$. However, Doebele et al. found that the miR-17-92 cluster inhibited angiogenesis and neovascularization in vitro and in vivo models ${ }^{[16]}$. Additionally, miR-93 has recently been identified as a negative regulator of VEGF in the kidneys under hyperglycemic conditions ${ }^{[17]}$. 
The role of miR-93 in the regulation of retinal angiogenesis still remains to be elucidated, and further studies investigating its function in the pathogenesis of ocular neovascular diseases could contribute to the development of novel therapies for these diseases. In this study, the laser-induced CNV mice model was used, which is a well-characterized animal model of exudative AMD, to confirm miR-93 involvement in CNV. The function of miR-93 and downstream targets were investigated in human microvascular endothelial cells (HMECs) as well.

\section{Materials and methods}

\subsection{Animals}

All experiments were performed in accordance with the ARVO Statement for the Use of Animals in Ophthalmic and Vision Research, and approved by Harbin Medical University and the Royal Victorian Eye and Ear Hospital Animal Research \& Ethics Committee. All mice were euthanized by $\mathrm{CO}_{2}$ inhalation and all efforts were made to minimize suffering.

\subsubsection{Establishing the mouse model of laser-induced $\mathrm{CNV}$ and in vivo transfection}

Adult C57BL/6 mice (9 weeks old) were maintained in a $12 \mathrm{~h}$ light/dark cycle with a room illuminance of 140-260 lux during the bright portion of the cycle. Animals were provided standard food and water ad libitum. Laser treatment was performed as described in a previous study ${ }^{[19]}$. CNV lesions were evaluated after laser treatment using micron III and choroidal flatmount. C57BL/6 mice were anesthetized through an intraperitoneal injection with a mixture of ketamine and xylazine $(100 \mathrm{mg} / \mathrm{kg}$ and $10 \mathrm{mg} / \mathrm{kg}$, respectively) in a dosage of 0.1 $\mathrm{mL} / 10 \mathrm{~g}$ of body weight. The pupils were dilated with a drop of $1 \%$ tropicamide solution (Alcon, USA), followed by $0.5 \%$ proxymetacaine hydrochloride (Alcon, USA) as a topical 
anaesthetic. Laser (50- $\mu \mathrm{m}$ spot size, $0.05 \mathrm{~s}$ duration, and $500 \mathrm{~mW})$ was applied using a laser photocoagulator (532-nm wavelength) to the retina radially about the optic nerve with a coverslip as a contact lens. The formation of a small bubble at the laser spot indicated a successful rupture of the Bruch's membrane and formation of CNV. Mice without the bubble formation or with vitreal haemorrhage obscuring the posterior segment were excluded. CNV lesions were evaluated after laser treatment using Micron III and histological staining of choroidal flatmount.

\subsubsection{Micron III Fundus imaging}

Anaesthetised mice pupils were dilated with a drop of $1 \%$ tropicamide solution (Alcon, USA). The mouse eyes were covered with a drop of ocular lubricating gel (Alcon, USA), which served as an optical coupling medium, and the vascular network of the live CNV mice were imaged in vivo using the Micron III retinal imaging camera system (Phoenix Research Laboratories, USA). Fundus images of both eyes were obtained before a dose of $1 \mathrm{~mL} / 10 \mathrm{~g}$ body weight of $1 \%$ fluorescein sodium was injected intraperitoneally into each mouse to obtain a fluorescence image.

\subsubsection{Choroidal flatmount}

To analyse the area of the CNV lesion, mice eyes were enucleated and fixed in $4 \%$ paraformaldehyde for $60 \mathrm{~min}$. Under a dissecting microscope the anterior segment and neuroretina were removed. The sclera-choroid-retinal pigment epithelial complex was isolated and incubated in a blocking solution $(0.5 \%$ bovine serum albumin, $0.5 \%$ triton-X) for $4 \mathrm{~h}$ at $4{ }^{\circ} \mathrm{C}$ and then for $16 \mathrm{~h}$ at $4^{\circ} \mathrm{C}$ in Isolectin GS-IB4 conjugated with Alexa Fluor 488 (1:100 dilution, $500 \mathrm{ug} / \mathrm{mL})$. Fluorescent micrographs of the choroidal flatmount were then taken using Olympus DP-71 camera system attached to an Olympus BX61 microscope. The 
area $\left(\mu \mathrm{m}^{2}\right)$ was measured using the ImageJ software. The measurements obtained from multiple lesions from eyes of each animal were averaged.

\subsubsection{Intravitreal injection of miRNA}

Scrambled mirVana ${ }^{\mathrm{TM}}$ miRNA Mimic, Negative Control \#1(Ambion, USA) or miR-93 mimic (Ambion, USA, 5'- CAAAGUGCUGUUCGUGCAGGUAG -3') at a concentration of $5 \mu \mathrm{M}$ was complexed with Invivofectamine 2.0 Transfection Reagent (Invitrogen, USA) in accordance with the manufacturer's instructions. The CNV mice were randomly divided into three groups ( $n=6 /$ group, and 12 eyeballs/group). Each group received either no treatment or an intravitreal injection of $5 \mu \mathrm{L}$ of either the scramble control miR or miR-93 mimic treatment on day 0 (immediately after laser treatment), which was readministered on day 7. For the intravitreal injection, a hole was first made by inserting a 30-gauge needle posterior to the limbus of the mice eye. Then the respective compounds were injected through the initial hole using a 34-gauge Hamilton syringe. Insertion and infusion were performed and directly viewed through an operating microscope. Care was taken not to injure the lens or the retina ${ }^{[16]}$. Mice were sacrificed on day 14 for choroidal flatmount, real-time quantitative polymerase chain reaction (qPCR), and Western blot analysis.

\subsection{Cell culture}

HMEC was obtained from Hitesh Peshavariya of Centre for Eye Research Australia. HMECs were grown in an endothelial basal medium (EBM, Lonza, Switzerland), supplemented with EGM-MV SingleQuot Kit Supplement \& Growth Factors (Lonza, Switzerland) at $37^{\circ} \mathrm{C}$ in a humidified chamber at $5 \% \mathrm{CO}_{2}$.

\subsubsection{In vitro transfection of HMECs}


A 10 nM mimicking miR-93 (Ambion, USA) or nonspecific control miRNA (Ambion, USA) was transfected into HMEC cell lines using Lipofectamine RNAiMAX (Invitrogen, USA) according to the manufacturer's instructions.

\subsubsection{Cell proliferation, migration and Tube formation assay}

After transfection, the cells were seeded into 96-well plates at $2 \times 10^{3}$ cells/well. The MTT assay kit (Roche Applied Science, USA) was used to determine the cell growth according to the manufacturer's instructions. The absorbance was measured at $570 \mathrm{~nm}$ with a reference wavelength of $690 \mathrm{~nm}$ using a microplate reader (Bio-Rad Benchmark Plus, USA).The ability of HMECs to form capillary-like tubes in culture was assessed by adding $5 \times 10^{4}$ cells to 200 $\mu \mathrm{L}$ of a pre-gelled Matrigel (BD Biosciences, USA) in $1 \mathrm{~mL}$ of a complete medium. Tube formation in the HMEC culture was quantified at $\mathrm{t}=9 \mathrm{~h}$ by counting all the loops formed in the gel under an inverted phase contrast microscope (Olympus IX71, Japan) at $\times 40$ magnification. The data was presented as the total number of loops formed. Migration of HMECs was monitored by generating a 'scratch' assay. After $48 \mathrm{~h}$ of transfection, $3 \times 10^{4}$ HMECs were plated in a 48-well plate previously labeled with lines to facilitate identification of the same regions at different time points. Cells were incubated for $48 \mathrm{~h}$ to reach confluence and a scratch was made in the monolayer using a $200 \mu \mathrm{L}$ pipette tip. Floating cells were removed by two gentle washes with PBS and the growth medium was replaced with EBM only. Reference photographs were taken using a phase contrast microscope (Olympus IX71, Japan) and the same areas photographed at $\mathrm{t}=9 \mathrm{~h}$. HMEC migration was quantified as the difference in size between the denuded area immediately after the scratch and at $\mathrm{t}=9 \mathrm{~h}$.

\subsection{RNA preparation and $q P C R$}


In mice, total mRNA was extracted using TRIzol (Invitrogen, USA) from Retinal Pigment Epithelium (RPE)/Choroids of both the control group and CNV group 14 days after the CNV laser procedure according to the user's manual. In cells, the total mRNA was isolated $48 \mathrm{~h}$ after transfection using the same method. The miR-93 (5' -

CAAAGUGCUGUUCGUGCAGGUAG-3') first-strand cDNA was synthesized using TaqMan MicroRNA reverse transcription kit (Applied Biosystems, USA). Quantitative qPCR was performed using the TaqMan MicroRNA assay (Applied Biosystems, USA), with U6 ( 5'-CGAGCACAGAATCGCTTCA-3' forward; 5'-CTCGCTTCGGCAGCACATAT-3' reverse) as the housekeeping gene. The qPCR analysis of mRNA was performed using HighC-apacity cDNA Reverse Transcription Kit (Applied Biosystems, USA) and TaqMan gene expression master mix (Applied Biosystems, USA) for VEGF-A (5'GTCTCGAGGGTTTCGGGAACCAGACCTCTCA-3’ forward; CAGAATTCCAGAAACAACCCTAATCTTCCGGG reverse).

18s (5'-GAAGGTGAAGGTCGGAGTC-3' forward; 5'-GAAGATGGTGATGGGATTTC3' reverse ) was used as the housekeeping gene. The reaction conditions were as follows: 2 min at $50^{\circ} \mathrm{C}$ followed by 45 cycles of $95^{\circ} \mathrm{C}$ for $10 \mathrm{~min}, 95^{\circ} \mathrm{C}$ for $15 \mathrm{~s}$ and $60{ }^{\circ} \mathrm{C}$ for $60 \mathrm{~s}$. The results were expressed as $\Delta \mathrm{Ct}$, and fold changes in relative mRNA expression were calculated using the equation $2^{-\Delta(\Delta \mathrm{Ct})}$. All assays were performed according to the manufacturer's instructions.

\subsection{Western blotting analysis}

The ocular tissues (RPE and choroid mix) and were isolated from enucleated eyes under dissecting microscope and homogenised in RIPA buffer containing a protease inhibitor cocktail (1:100 dilution, Sigma-Aldrich, USA). Cells were collected and lysed as above using 
RIPA buffer. The lysates were cleared by centrifugation at $12,000 \mathrm{~g}$ for $20 \mathrm{~min}$ at $4^{\circ} \mathrm{C}$. The protein concentration of the lysate was determined using the bicinchoninic acid (BCA) assay. Equal amounts of proteins ( $30 \mu \mathrm{g}$ in vivo and $20 \mu \mathrm{g}$ in vitro) were separated by SDS-PAGE gels (Invitrogen, USA) and transferred onto polyvinylidene fluoride (PVDF) membranes (GE Healthcare, UK). After blocking with 5\% nonfat milk powder in TBS (pH 7.5), the membranes were incubated with anti-VEGF-A (ab51745, Rabbit polyclonal to VEGF-A, Abcam Sapphire Bioscience, USA) and anti- $\beta$-actin (ab3280, Mouse monoclonal to Actin, Abcam Sapphire Bioscience, USA). After incubation with the HRP-conjugated secondary IgG (1:10,000, Sigma, USA), blots were developed and quantified with Odyssey Infrared Imaging System (Li-Cor Biosciences, USA).

\subsection{Statistical analysis}

All values were shown as mean \pm SD. Statistical significance between the groups was assessed using either analysis of ANOVA or two tailed t-test, as appropriate, by the Prism 6.0 software (GraphPad Software, USA). For all statistical tests, a $\mathrm{p}<0.05$ was considered statistically significant.

\section{Results}

\subsection{Expression of miR-93 in the laser-induced CNV mouse model}

The expression of miR-93 in mice eyes was measured by qPCR (RPE/Choroid mix $n=6$ ). miR-93 expression was significantly downregulated $(\mathrm{p}<0.01)$ in the CNV eyes compared with the untreated eyes (Fig. 1). CNV mice were transfected using a transfection agent coupled with either a miR-93 mimic or a negative control oligonucleotide (miR-control). The successful miR-93 transfection was confirmed using PCR analysis $(n=6, p<0.01)$ after 
injection with miR-93 mimic (Fig. 1). We demonstrated that the miR-93 remains effective even after 14 days of in vivo transfection.

\subsection{VEGF-A expression in the CNV mice eyes}

The downstream effect of reduced miR-93 expression was assessed by qPCR and Western blot analysis. The expression of VEGF-A, a potent stimulator of blood vessel growth and predicted target of miR-93, was found to be significantly upregulated in CNV mice ( $\mathrm{n}=6$, $\mathrm{p}<0.05$ ) compared to untreated controls (Fig. 2). Similarly, an increase in VEGF-A protein levels $(n=6, p<0.05)$ was observed in CNV mice compared with untreated ones (Fig. 3). To further explore the effects of miR-93 in vivo, the expression of miR-93 was altered in the CNV mice to determine if restoration of miRNA expression would result in modulation in the expression of predicted mRNA and protein targets. A transfection agent was coupled to either a mir-93 mimic or a negative control oligonucleotide, and transfected by an intravitreal injection into the eyes of the CNV mice after laser treatment.

CNV mice with overexpressed miR-93 exhibited significant decrease in mRNA and protein expression of VEGF-A $(n=6, p<0.05)$, measured by qPCR and Western blot, respectively (Figs. 2 and 3). A clear functional impact of miR-93 regulated downstream targets on CNV severity in vivo has been demonstrated.

\subsection{Overexpression of miR-93 in HMECs reduced VEGF-A expression and proliferation of HMECs.}

After successful transfection of miR-93 in HMECs (Fig. 4), the mRNA and protein expression of VEGF-A was significantly reduced in cells overexpressing miR-93 compared with control cells $(\mathrm{p}<0.05)$ (Fig. 5). 
miR-93 regulated cell function was determined in miR-93 overexpressed HMECs. We did MTT, tube formation and scratch assay after HMECs received scramble control miR or miR93 mimic treatment. The MTT assay showed a significant decrease $(n=4, p<0.01)$ in the miR93 transfected HMECs compared to both the nontransfected and miR-control groups (Fig. 6 A). However, there no significant changes were found in the tube formation and the migration activity of cells determined by the scratch assay (Fig. $6 \mathrm{~B}$ and C).

\subsection{Effect of miR-93 transfection on the CNV lesions in mice eyes}

CNV lesion size and severity were determined to confirm the effect of the overexpression of miR-93 on the CNV mice. Representative composite fundus images taken 1-2 weeks posttreatment were shown in Fig. 7. The findings of this study demonstrated that transfection with the negative control miR showed no changes in CNV lesion area measurements of choroidal flatmount ( $\mathrm{n}=6, \mathrm{P}>0.05$, Fig. $8 \mathrm{~A}$ ) but the mice eyes treated with miR-93 mimics had significantly smaller CNV lesion areas $(n=6, P<0.01$, Fig. 8A), demonstrating that miR-93 successfully reduced angiogenesis in the mice eyes (Fig. 8B-D).

\section{Discussion}

Since the identification of miRNAs as a distinct class of biological regulators in the early 2000, numerous miRNAs have been identified in humans ${ }^{[18]}$. One miRNA can target multiple mRNA, and a single mRNA can be targeted by several miRNAs ${ }^{[21,27,15]}$. Many studies have revealed that miRNAs play crucial roles in many diseases and can serve as potential therapeutics. Angiogenic factors have been implicated in severe neovascular pathologies, including diabetes mellitus ${ }^{[20,40]}$ and tumor vascularization ${ }^{[16,38]}$. However, the role of miRNA's on ocular neovascular disorders remains unclear. Identification of particular miRNA's, or miRNA clusters involved in choroidal neovascularization would both further 
our knowledge of the complex regulatory and dynamic mechanisms involved in exudative AMD but may also identify future therapeutic targets.

Several miRNA candidates have been identified in ocular neovascularization diseases ${ }^{[29]}$. Mulik et al. demonstrated 10 to 20 fold increase of miR-132 expression in severe corneal neovascularization after herpes simplex virus infection ${ }^{[26]}$; miR-200b has been shown to regulate angiogenesis in both STZ-induced diabetic rats and diabetic human eyes ${ }^{[25]}$. miR-93 has a predicted complementary interaction with VEGF-A and can regulate VEGF in the kidneys of diabetic $\mathrm{db} / \mathrm{db}$ mouse model ${ }^{[24]}$. We proposed that miR-93 may play a potential role in CNV through down regulation of VEGF.

Despite a predicted interaction with the 3' UTR VEGF-A, literature support for miR-93's regulation of angiogenesis is limited. Moreover, findings on the role of miR-93 tend to be contradictory. miR-93's reported role in breast and colorectal tumorogenesis is to increase cell migration, angiogenesis and promot tumor formation; such that miR-93 and its parent cluster has been labelled as 'oncomirs, ${ }^{[7-9]}$. However, Xiao et al. have observed significant downregulation of miR-93 in human samples of advance stage colon tumoral tissue ${ }^{[36,39]}$ and Yu et al. demonstrated a suppression proliferation of human colon cancer stem cells. We would like to know whether miR-93 is a promoter or inhibitor of CNV in the eye.

In our study, miR-93 was demonstrated to suppress CNV formation and severity. Reduced expression of miR-93 was observed in the experimental laser-induced CNV mice model (Fig. 1), inferring miR-93 involvement in the formation of CNV complex. Laser induced CNV through rupture of the Bruch's membrane is currently the most widely utilized model of neovascular AMD. This model replicates the unique and complex pathology of wet AMD reliably, with respect to release of angiogenic factors, neovascularization and inflammatory 
response. Our in vivo data suggests miR-93 regulates angiogenesis at least in part through regulation of VEGF-A. Restoration of miR-93 in vivo mitigates VEGF expression (Fig. 2 and 3 respectively) and significantly reduces the severity of CNV (Fig. 7 and 8). These observations are consistent with other studies that reported a downregulation of miR-93 in retinas of diabetic animal models, where significant pathological ocular neovascularisation is involved $^{[21]}$.

Kamal et al. suggested that HMECs are an appropriate model for studies involving microvascular endothelial cells such as angiogenesis ${ }^{[12]}$. Costa et al. used HMECs to compare the multiple effects of bevacizumab and ranibizumab in ocular angiogenesis, they indicated that HMEC is probably a more accurate model to study the effects on the angiogenic process, given their capillary background, characteristic of angiogenic vessels ${ }^{[4]}$. HMECs were used here as the in vitro replication of the microvasculature resident in the eye. Similar to the in vivo results, the overexpression of miR-93 of cultured HMECs resulted in a significant reduction in both mRNA and protein expression of VEGF, and a significant decrease in HMEC proliferation (Fig. 5 and 6 respectively).

Our findings that miR-93 suppress angiogesis through key potent angiogenic factors differs from the previous reports showing that miR-93 is pro-angiogenic. Shen et al. demonstrated that miR-31, miR-150 and miR-184 were down-regulated in a mouse model of oxygeninduced retinopathy ${ }^{[27]}$ whereas Zhuo et al. demonstrated an up-regulation of the miR23 24 27 cluster which support the notion of miRNAs regulation of vascular development in the retina ${ }^{[35]}$. The group of Ghosh found that miR-424 promotes angiogenesis inhibiting cullin 2 (CUL2) and thereby increasing HIF-1 a levels ${ }^{[10]}$. miR-22 was found to decrease its expression in human colon cancer, and acted as anti-angiogenic factor through inhibiting HIF-1 a expression ${ }^{[37]}$. miR-497 expression was down-regulated in human ovarian cancer 
tissues, which was significantly associated with increased angiogenesis ${ }^{[34]}$. Liu et al. indicated that miR-21 can induce tumor angiogenesis through targeting PTEN in human prostatic cancer cells $^{[23]}$. miRNAs have been shown to act in a cell type-specific manner. miRNAs regulate gene expression through interaction with specific promoters or integration into introns of protein encoding genes ${ }^{[22,28]}$. The eye is a unique organ with blood-ocular barriers, which limit influence of systemic factors and could account for the local variation of the miR-93 levels in the ey ${ }^{[13]}$. This may also account for the inconsistencies of miR-93 role in pathogenic mechanisms of ocular diseases. The results from this study highlight a need for further exploration into miRNAs as their multivariate pathways and target interactions contribute significantly to pathogenesis.

Identification of the regulatory roles of miR-93 could have significant implications in future avenues of treatment for exudative AMD and other ocular pathologies with microvascular complications. Although most candidates are in preclinical phases, significant progress has been made in human trials for treating of hepatitis $\mathrm{C}$ and liver cancer ${ }^{[1]}$. The potential of miR93 as a therapeutic target is promising and should be further explored for the treatment of AMD.

\section{Acknowledgement}

We would like to thank the staff of EMSU for their assistance with animal care.

\section{References}

1. Bouchie A (2013) First microRNA mimic enters clinic. Nat Biotechnol 31:577

2. Campochiaro PA, Nguyen QD, Shah SM, Klein ML, Holz E, Frank RN, Saperstein DA, Gupta A, Stout JT, Macko J, DiBartolomeo R, Wei LL (2006) Adenoviral vector- 
delivered pigment epithelium-derived factor for neovascular age-related macular degeneration: results of a phase I clinical trial. Hum Gene Ther 17:167-1763

3. Caporali A, Emanueli C (2011) MicroRNA regulation in angiogenesis. Vascul Pharmacol 55:79-86

4. Costa R, Carneiro A, Rocha A, Pirraco A, Falcão M, Vasques L, Soares R (2009) Bevacizumab and ranibizumab on microvascular endothelial cells: A comparative study. J Cell Biochem 108: 1410-1417

5. Chen Y, Gorski DH (2008) Regulation of angiogenesis through a microRNA (miR-130a) that down-regulates antiangiogenic homeobox genes GAX and HOXA5. Blood 111: $1217-1226$

6. Doebele C, Bonauer A, Fischer A, Scholz A, Reiss Y, Urbich C, Hofmann WK, Zeiher AM, Dimmeler S (2010) Members of the microRNA-17-92 cluster exhibit a cell-intrinsic antiangiogenic function in endothelial cells. Blood 115: 4944-4950

7. Du L, Zhao Z, Ma X, Hsiao TH, Chen Y, Young E, Suraokar M, Wistuba I, Minna JD, Pertsemlidis A (2014) miR-93-directed downregulation of DAB2 defines a novel oncogenic pathway in lung cancer. Oncogene 33:4307-4315

8. Fang L, Deng Z, Shatseva T, Yang J, Peng C, Du WW, Yee AJ, Ang LC, He C, Shan SW, Yang BB (2011) MicroRNA miR-93 promotes tumor growth and angiogenesis by targeting integrin-beta8. Oncogene 30:806-821

9. Fang L, Du WW, Yang W, Rutnam ZJ, Peng C, Li H, O'Malley YQ, Askeland RW, Sugg S, Liu M, Mehta T, Deng Z, Yang BB (2012) MiR-93 enhances angiogenesis and metastasis by targeting LATS2. Cell Cycle 11:4352-4365 
10. Ghosh, G., Subramanian, I.V., Adhikari, N., Zhang, X., Joshi, H.P., Basi, D., Chandrashekhar, Y.S., Hall, J.L., Roy, S., Zeng, Y., Ramakrishnan, S (2010) Hypoxiainduced microRNA-424 expression in human endothelial cells regulates HIF-alpha isoforms and promotes angiogenesis. J. Clin. Invest 120: 4141-4154

11. Grossniklaus HE, Kang SJ, Berglin L (2010) Animal models of choroidal and retinal neovascularization. Prog Retin Eye Res 29:500-519

12. Kamal K, Du W, Mills I, Sumpio BE (1998) Antiproliferative effect of elevated glucose in human microvascular endothelial cells. Journal of cellular biochemistry 71: 491-501

13. Kramer AaWB-B (2002) Antiseptic Prophylaxis and Therapy in Ocular Infections: Principles, Clinical Practice and Infection Control. Longman, New York

14. Klein R, Klein BE, Linton KL (1992) Prevalence of age-related maculopathy.The Beaver Dam Eye Study. Ophthalmology 99:933-943

15. Krek A, Grün D, Poy MN, Wolf R, Rosenberg L, Epstein EJ, MacMenamin P, da Piedade I, Gunsalus KC, Stoffel M, Rajewsky N (2005) Combinatorial microRNA target predictions. Nat Genet 37:495-500

16. Kuehbacher A, Urbich C, Dimmeler S (2008) Targeting microRNA expression to regulate angiogenesis. Trends Pharmacol Sci 29:12-15

17. Kwak N, Okamoto N, Wood JM, Campochiaro PA (2000) VEGF is major stimulator in model of choroidal neovascularization. Invest Ophth Vis Sci 41:3158-3164

18. Lagos-Quintana M, Rauhut R, Lendeckel W, Tuschl T (2011) Identification of novel genes coding for small expressed RNAs. Science 294:853-858 
19. Lambert V, Lecomte J, Hansen S, Blacher S, Gonzalez ML, Struman I, Sounni NE, Rozet E, de Tullio P, Foidart JM, Rakic JM, Noel A (2013) Laser-induced choroidal neovascularization model to study age- macular degeneration in mice. Nat Protoc $8: 2197-2211$

20. Leeper NJ, Cooke JP (2011) MicroRNA and mechanisms of impaired angiogenesis in diabetes mellitus. Circulation 123:236-238

21. Lewis BP, Shih IH, Jones-Rhoades MW, Bartel DP, Burge CB (2003) Prediction of mammalian microRNA targets. Cell 115:787-798

22. Ling H, Fabbri M, Calin GA (2013) MicroRNAs and other non-coding RNAs as targets for anticancer drug development. Nature Reviews Drug Discovery 12:847-865

23. Liu LZ, Li C, Chen Q, Jing Y, Carpenter R, Jiang Y, Kung HF, Lai L, Jiang BH (2011) MiR-21 induced angiogenesis through AKT and ERK activation and HIF-1alpha expression. PLoS ONE 6: e19139

24. Long J, Wang Y, Wang W, Chang BH, Danesh FR (2010) Identification of microRNA93 as a novel regulator of vascular endothelial growth factor in hyperglycemic conditions. J Biol Chem 285:23457-23465

25. McArthur K, Feng B, Wu Y, Chen S, Chakrabarti S (2011) MicroRNA-200b regulates vascular endothelial growth factor-mediated alterations in diabetic retinopathy. Diabetes 60:1314-1323

26. Mulik S, Xu J, Reddy PB, Rajasagi NK, Gimenez F, Sharma S, Lu PY, Rouse BT (2012) Role of miR-132 in angiogenesis after ocular infection with herpes simplex virus. Am J Pathol 181:525-534 
27. Rajewsky N, Socci ND (2004) Computational identification of microRNA targets. Dev Bio 267:529-535

28. Roberts TC. The MicroRNA Biology of the Mammalian Nucleus (2014) Mol Ther Nucleic Acids 3:e188

29. Saxena K, Rutar MV, Provis JM, Natoli RC (2014) Identification of miRNAs in a Model of Retinal Degenerations. Invest Ophthalmol Vis Sci 56:1820-1829

30. Schlenker MB, Thiruchelvam D, Redelmeier DA (2015) Intravitreal anti-vascular endothelial growth factor treatment and the risk of thromboembolism. Am J Ophthalmol $160: 569-580$

31. Shen J, Yang X, Xie B, Chen Y, Swaim M, Hackett SF, Campochiaro PA. (2008) MicroRNAs regulate ocular neovascularization. Mol Ther 16:1208-1216

32. Siemerink MJ, Klaassen I, Van Noorden CJ, Schlingemann RO (2013) Endothelial tip cells in ocular angiogenesis: potential target for anti-angiogenesis therapy. J Histochem Cytochem 61:101-115

33. van Romunde SH, Polito A, Bertazzi L, Guerriero M, Pertile G (2015) Long-Term Results of Full Macular Translocation for Choroidal Neovascularization in Age-Related Macular Degeneration. Ophthalmology 122:1366-1374

34. Wang W, Ren F, Wu QH, Jiang DZ, Li HJ, Shi HR (2014) MicroRNA-497 suppresses angiogenesis by targeting vascular endothelial growth factor A through the PI3K/AKT and MAPK/ERK pathways in ovarian cancer. Oncol. Rep 32: 2127-2133 
35. Wong WL, Su X, Li X, Cheung CM, Klein R, Cheng CY, Wong TY (2014) Global prevalence of age-related macular degeneration and disease burden projection for 2020 and 2040: a systematic review and meta-analysis. Lancet Global Health 2:106-116

36. Xiao ZG Deng ZS, Zhang YD, Zhang Y, Huang ZC (2013) Clinical significance of microRNA-93 downregulation in human colon cancer. Eur J Gastroenterol Hepatol 25:296-301

37. Yamakuchi M, Yagi S, Ito T,Lowenstein CJ (2011) MicroRNA-22 regulates hypoxia signaling in colon cancer cells. PLoS ONE 6: e20291.

38. Yang F, Wang W, Zhou C, Xi W, Yuan L, Chen X, Li Y, Yang A, Zhang J, Wang T (2015) MiR-221/222 promote human glioma cell invasion and angiogenesis by targeting TIMP2.Tumour Biol 36:3763-3773

39. Yu XF, Zou J, Bao ZJ, Dong J (2011) miR-93 suppresses proliferation and colony formation of human colon cancer stem cells. World J Gastroenterol 17:4711-4717

40. Zampetaki A, Kiechl S, Drozdov I, Willeit P, Mayr U, Prokopi M, Mayr A, Weger S, Oberhollenzer F, Bonora E, Shah A, Willeit J, Mayr M (2010) Plasma microRNA profiling reveals loss of endothelial miR-126 and other microRNAs in type 2 diabetes. Circ Res 107:810-817

41. Zhou Q, Gallagher R, Ufret-Vincenty R, Li X, Olson EN, Wang S (2011) Regulation of angiogenesis and choroidal neovascularization by members of microRNA-23 27 24 clusters. Proc Natl Acad Sci USA 108:8287-8292 


\section{Figures Legends}

\section{Figure 1. miR-93 expression in CNV mice}

Expression of miR-93 in CNV mice in the following treatment groups:

(1) No laser-induced choroidal neovascularization (no CNV): Control mice without treatment.

(2) CNV: Mice exposed to CNV laser treatment only, without transfection.

(3) $\mathrm{CNV}+$ miR control: Mice exposed to CNV laser treatment and transfected with negative control oligonucleotides via intravitreal injection.

(4) CNV + miR-93: Mice exposed to CNV laser treatment and transfected with miR-93 mimics via intravitreal injection.

Data are presented as relative fold change of miR-93 $\pm \mathrm{SD}, \mathrm{n}=6,{ }^{*} \mathrm{p}<0.05, * * \mathrm{p}<0.01$.

\section{Figure 2. Expression of VEGF-A in CNV mice after transfection}

VEGF-A mRNA expression in eye tissue of control mice and CNV mice either transfected with a miR control or a miR-93 mimic via intravitreal injection. Data are presented as mean fold change of mRNA levels $\pm \mathrm{SD}, \mathrm{n}=6, * * \mathrm{p}<0.01$.

\section{Figure 3. Protein expression of VEGF-A in mice eyes}

(A) Western Blot images of VEGF-A protein in mice eyes of different groups: no CNV; CNV only; CNV mice transfected with miR-Control or CNV mice transfected with miR-93. 
(B) Quantification of relative VEGF-A protein expression in no CNV, CNV and different group of CNV mice after transfection. Data are presented as relative VEGF-A protein expression $\pm \mathrm{SD}, \mathrm{n}=6,{ }^{*} \mathrm{p}<0.05$.

\section{Figure 4. Expression of miR-93 in HMECs}

miR-93 expression in HMECs without transfection or transfected with either negative control or miR-93. Data are expressed as relative fold change of miR-93 $\pm \mathrm{SD}, \mathrm{n}=4, * * \mathrm{p}<0.01$.

\section{Figure 5. Expression of VEGF-A in HMECs}

(A) VEGF-A mRNA expression in HMECs transfected with both miR control and miR-93 mimics. Data are expressed as mean fold change to miR-93 levels $\pm \mathrm{SD}, \mathrm{n}=4,{ }^{* *} \mathrm{p}<0.01$.

(B) Expression pattern of the target proteins in HMECs after transfection of either the negative control miR-93 measured by Western blot.

(C) Quantification of relative VEGF-A protein expression in HMECs receiving miR control or miR-93 transfection determined by Western blot. Data are presented as mean $\pm \mathrm{SD}$, $\mathrm{n}=4 /$ group, $* * \mathrm{p}<0.01$.

\section{Figure 6. HMECs proliferation, Tube formation and migration}

(A) Proliferative activity of HMECs was transfected with either a negative control or miR-93 mimic. Data are expressed as mean relative absorbance level $\pm \mathrm{SD}, \mathrm{n}=4, * * \mathrm{p}<0.01$.

(B) Analysis of capillary-like tube formation of HMECs with no transfection, miR control and miR-93 mimic. Data are presented as mean number of loops formed \pm SD n=4, $>0.05$.

(C) Migration of HMECs. The graphs show the mean migrated distance $(n m) \pm S D n=4, p>$ 0.05 . 


\section{Figure 7. Color fundus image of CNV mice}

(A) A representative color fundus image of the mouse after laser-induced CNV treatment. (B) FFA of CNV lesion of mouse receiving CNV generation only. (C) FFA of mouse receiving CNV generating laser treatment and miR-Control oligonucleotide. (D) FFA of mouse receiving CNV generating laser treatment and miR-93 mimic.

\section{Figure 8. CNV mice lesion areas and flatmount}

A: Quantification of relative 2D lesion areas in the eyes of CNV mice after laser treatment in choroidal flatmount. Lesion areas were measured using ImageJ. Data are expressed as relative $\mathrm{CNV}$ lesion area compared to $\mathrm{CNV}$ only group $\pm \mathrm{SD}, \mathrm{n}=6,{ }^{*} \mathrm{p}<0.05$.

B-D: Representative micrographs of CNV lesions of Isolectin GS-IB4 *AF488 stained choroidal flatmounts, with manual border delineation in ImageJ.

B: CNV only; C: CNV+miR control; D:CNV+miR-93. 


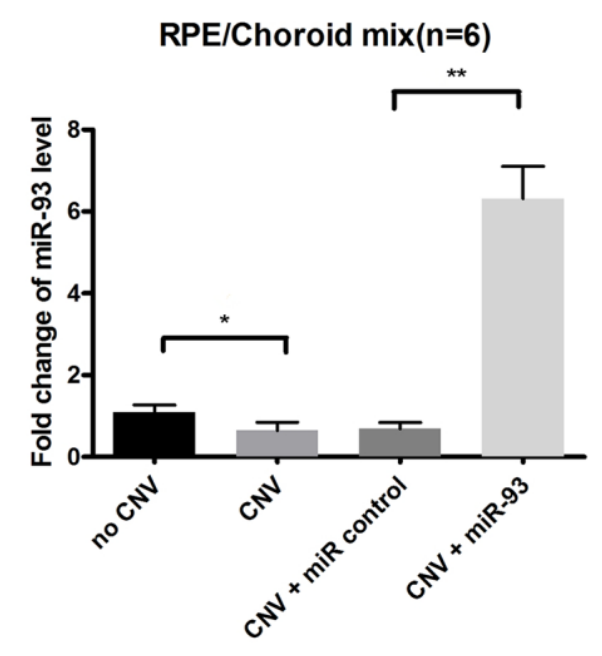




\section{RPE/Choroid mix $(n=6)$}

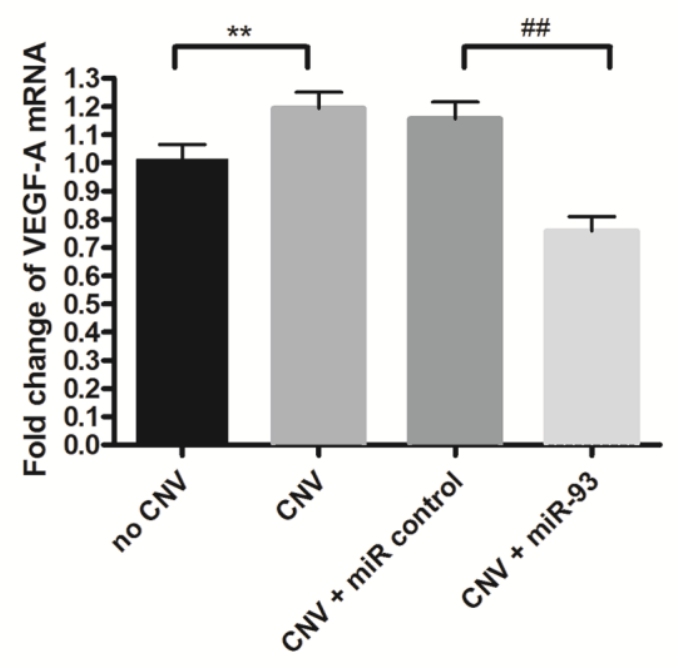


RPE/Choroid mix $(n=6)$

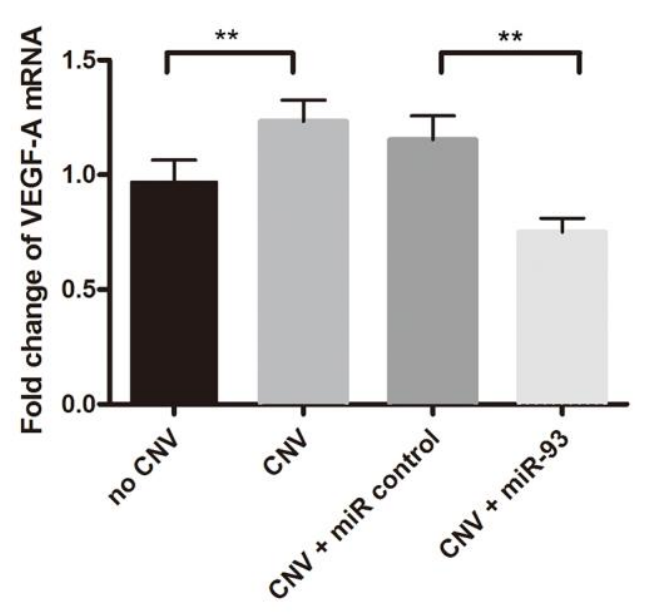


A

CNV mice western blot

B

RPE/Choroid mix (n=6)

B-actin 42 kDa

VEGF-A

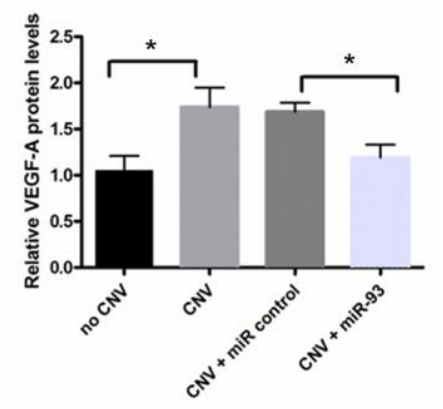


HMEC with and without transfection( $n=4)$

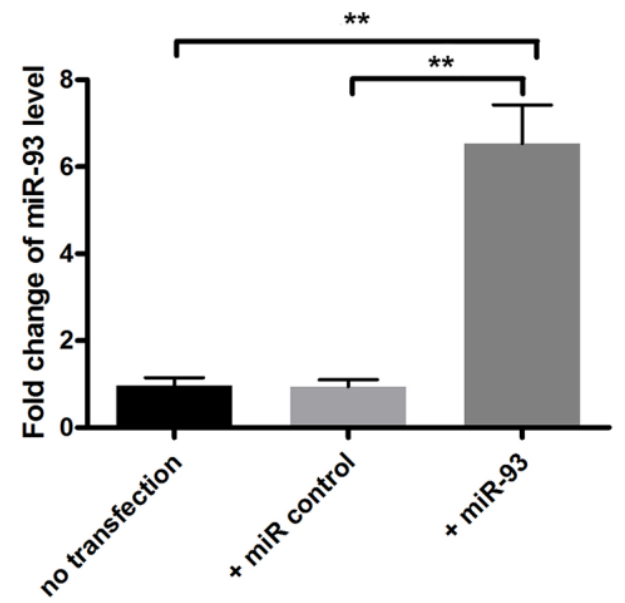




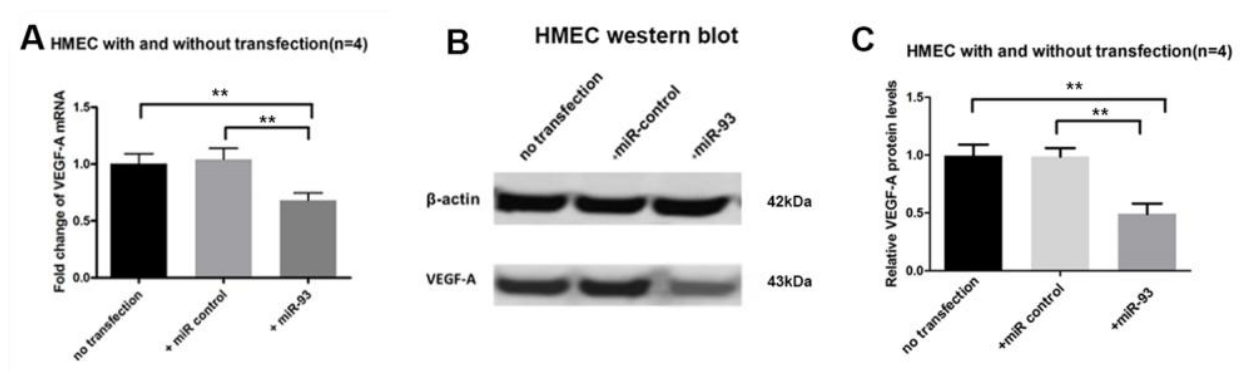


A HMEC with and without transfection MTT $(n=4)$

B HMEC with and without transfection Tube formation $(n=4) \quad$ C HMEC with and without transfection Migration( $n=4)$
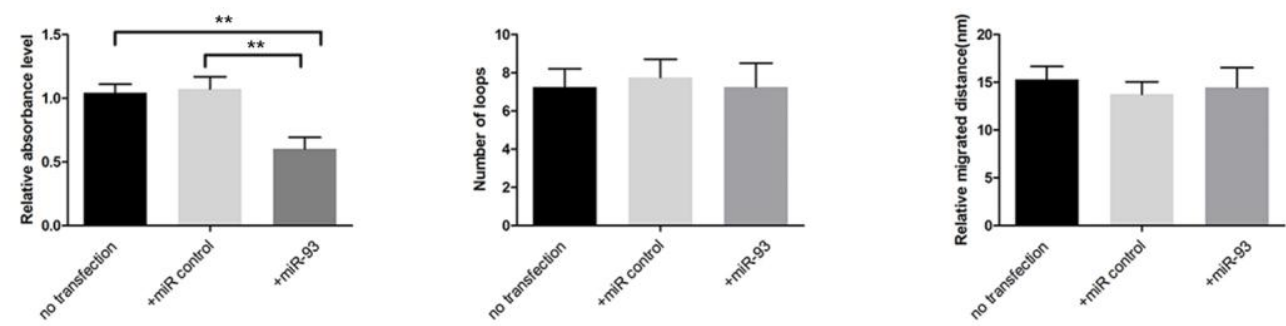


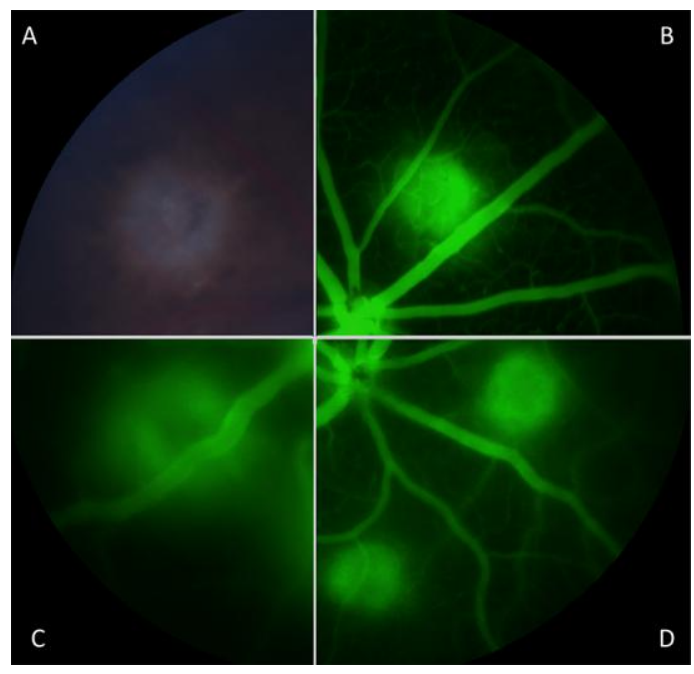

miR-93 in AMD 
A CNV with and without transfection lesion area( $(n=6)$

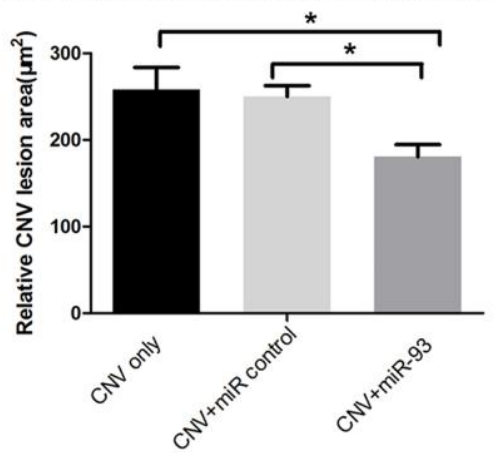

B C D
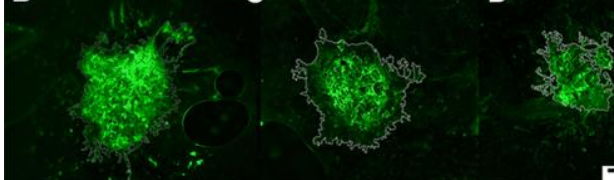

$\sin 20$

$-20 \overline{m m}$ 


\section{University Library}

\section{- M M N E R VA A gateway to Melbourne's research publications}

Minerva Access is the Institutional Repository of The University of Melbourne

Author/s:

Wang, L;Lee, AYW;Wigg, JP;Peshavariya, H;Liu, P;Zhang, H

Title:

miRNA involvement in angiogenesis in age-related macular degeneration

Date:

2016-12-01

Citation:

Wang, L., Lee, A. Y. W., Wigg, J. P., Peshavariya, H., Liu, P. \& Zhang, H. (2016). miRNA involvement in angiogenesis in age-related macular degeneration. JOURNAL OF PHYSIOLOGY AND BIOCHEMISTRY, 72 (4), pp.583-592. https://doi.org/10.1007/ s13105-016-0496-2.

Persistent Link:

http://hdl.handle.net/11343/282899 